\title{
Oscillation processes as a tool of physics cognition
}

\author{
Alexander A. Antonov ${ }^{1 *}$ \\ ${ }^{1}$ Research Centre of Information Technologies "TELAN Electronics" of the IAOI \\ * Correspondence author: Tel/fax (380) 444243587; e-mail: telan@bk.ru \\ ABSTRACT
}

\begin{abstract}
Based on research of resonant and transitional processes in electric LC-, RL-, RC-, and LCRcircuits, the article offers theoretical and experimental proof of physical reality of complex frequency and other concrete complex numbers which are a function of complex frequency. Analysis of resonant processes in electric circuits has shown inconsistency in their conventional interpretation at real frequencies. On the contrary, interpretation of resonance at complex frequencies allowed explaining all the inconsistencies of its interpretation at real frequencies. We provide the description of experiments confirming physical reality of resonance at complex frequencies. As for transitional processes in electric circuits, their analysis by means of differential equations requires the solution of the respective characteristic algebraic equations. In this respect we demonstrate that the solution of algebraic equations with the use of real numbers rules out the existence of oscillation transitional process, and, therefore, does not conform to physical reality.
\end{abstract}

Keywords: electric circuits, resonance, transitional processes, real frequencies, complex frequencies.

\section{INTRODUCTION}

It is well-known that in order to learn and comprehend new knowledge, it is necessary:

for the new knowledge to be based on a solid foundation of the existing knowledge (this refers to the reader),

for the new knowledge to lack inherent inconsistency (this refers to the author of a published work).

However, these requirements are not always met. Knowledge contained in textbooks is not absolutely true. Sooner or later it becomes obsolete and has to be reviewed. This is normal.

What is not normal is that sometimes the material in textbooks is presented in a way suggesting that the corresponding science already knows everything. And what does not correspond to this dogma is carefully concealed from the reader.

This approach does not take into account the very important fact that the objective of academic and scientific literature is not only to give the reader some particular knowledge, but also to teach the reader to think. To this end, it is necessary to give the reader some food for thought. Readers need to know what issues of the current scientific views are questionable, what the alternative explanations of certain phenomena are, and what facts cannot be given a scientific explanation so far.

With this in mind, let us try to discuss complex numbers.

Numbers - understandable and not understandable: We live in the world of numbers, various numbers having various meaning. We always have to count, estimate, and make decisions, we constantly make choices.

Thereupon, let us first of all remember that numbers can be dimensional (or concrete), and nondimensional. Concrete, or dimensional, numbers are numbers having a dimension, i.e. supplied with an indicator of measurement unit, for instance, 2 meters, 2 kilos, 2 cars, 2 hours, etc. In fact, all these numbers belong to the physically real world we live in. What about the non-dimensional numbers? Firstly, these numbers represent the ratios of concrete numbers having the same dimension, e.g., percentage. Secondly, here belong all numbers used in mathematics, as this way they are easier to use.

Furthermore, as is well-known, numbers can be positive and negative. Their meaning is easy to understand. For instance, in terms of geometry, if a positive number corresponds to a distance in a certain direction, then a negative number corresponds to the same distance in the opposite direction. Similarly, the weight of $-2 \mathrm{~kg}$ corresponds to the force opposite in its direction to the gravity force of $2 \mathrm{~kg}$, and may be, for instance, the so called lifting force allowing balloons to float. And in terms of money $-\$ 100$ means that you owe someone the $\$ 100$.

The use of geometric images also allows easily explaining the meaning of integer, fractional, and irrational numbers, although irrational numbers may 
raise some doubt, like, e.g. $\sqrt{2}, \sqrt{5}$ etc. Why are they an unending decimal, although this precision is never required in everyday life? Pictures of rightangle triangles having sides of the corresponding value allow explaining the situation.

As is seen, the most understandable explanations require the use of geometric images. Their particular importance in mathematics is accounted for by the fact that people think mostly with visual images.

All the previously discussed understandable numbers belong to the category of real numbers.

However, there are also the completely incomprehensible, the so called imaginary numbers, obtained by multiplying real numbers by an imaginary

unit $\sqrt{-1}=i$, as well as complex numbers which are a sum of real and imaginary numbers. There are also even more complicated, the so-called hyper-complex numbers (Kantor \& Solodovnikov, 1989), which we, however, will not discuss in this report. As for imaginary numbers, they were first discovered over 500 years ago. To be exact, G. Cardano discovered them in 1545, and R. Bombelli first used imaginary numbers to solve cubic equations in 1572. Since then, a complete mathematical theory of complex variable function has been developed (Markushevich 2005; Kreyszig 2006; Shaw 2006), which is widely used in applied science - radio electronics, optics, telecommunication, etc.

However, the meaning of imaginary and complex numbers in terms of simple common sense is still unclear. For instance, I. Newton (1642 - 1727) did not include them into the concept of number at all (Vinogradov 1982). Contemporary physicists do the same, and this is understandable. If complex numbers are admitted to exist, their physical meaning will have to be explained.

But sooner or later this has to be done. And, apparently, it is just about time to start.

Oscillations - understandable and not understandable: We live in the world of oscillations, and these oscillations are very different. We hear due to acoustic oscillations. We see because of electromagnetic oscillations. We warm by the fire and get $\tan$ on the beach due to electromagnetic oscillations, as well. There are also sea waves, high and low tides, seasonal changes of weather, economic crises, rotary motions in various devices, hurricanes, etc. All these phenomena have been known for a long time, and they have the relevant mathematical descriptions. This is why they seem to be quite understandable.
However, a more detailed study reveals some not understandable peculiarities of oscillation processes. For instance, resonance is one of the most wellknown and deeply researched oscillation processes. Resonance processes originating irrespective of our will - for instance, flutter in aviation - can have disastrous consequences. On the contrary, resonance processes which are well studied and used intentionally - e.g. in various radio-electronic filters - have huge benefits. We can even argue that contemporary communication technology would be impossible without the use of resonance.

Therefore, it is natural that resonance has been researched in depth in applied sciences, including the theory of electric circuits (Bode 1945; Balabanian 1994; Franko 1994; Cogdell 1997;Edminister \& Nahvi 1997; Raymond\&Pen-Min2001;Steinmetz 2006; Bell 2009).

Analysis of resonance in electric circuits widely uses complex numbers complex resistances, complex conductivities, complex voltages, complex frequencies, etc. The theory of electric circuits uses research tools based on real frequencies, as well. Both methods yield similar results, but not the same.

For instance, when analyzing immitance (e.g. transfer) functions of electric L,C-circuits, real frequencies at which the immitance function takes zero and infinite values are referred to as resonance frequencies. However, when analyzing immitance (e.g. transfer) functions of electric L,C,R-circuits it turns out that they do not take either zero or infinite modulo values at any value of real frequencies. Therefore, in electric L,C,R-circuits resonant frequencies imply the frequencies at which the immitance function takes extreme values.

Analysis of electric L,C-circuits, as well as L,C,Rcircuits at resonance frequencies has shown that their immitance functions can take zero value at some values of complex frequency, and can take infinitely large modulo value at other values of complex frequency. However, these frequencies are usually referred to not as resonance, but, respectively, as 'zeros' and 'poles'. This doesn't make any sense either, does it?

However, sometimes they are indeed referred to as resonance complex frequencies (Dolginov 1957; Antonov 1987).

Moreover, precise analysis (contrary to the commonly used simplified analysis) of electric L,C,R-circuits has shown that they actually have not a single, but a number of various real resonance frequencies (Antonov 2008; Antonov 2009). Furthermore, different electric L,C,R-circuits of the same order (e.g. 
second-order for the simplest oscillation loop) turn out to have a different number of these various real resonance frequencies. In addition, it also turned out that the real frequency of free oscillations in electric L,C,R-circuits, unlike electric L,C-circuits, never equals to any of the real resonance frequencies. The latter circumstance was pointed to in the first half of the $20^{\text {th }}$ century by academician Mandelstam (Mandelshtam 1955).

Thus, we can state that interpretation of resonance at real frequencies is inherently inconsistent, and, therefore, unsatisfactory.

However, it all becomes simple and clear if we conduct the analysis of electric circuits at complex frequencies. For electric L,C- and L,C,R-circuits of the same order, for any configuration, we always get one and the same number of complex resonance frequencies, which, moreover, coincide with complex frequencies of free oscillations.

So why the terms 'zeros' and 'poles' are nevertheless used in most publications, instead of the term 'complex resonance frequencies'? The answer is simple - if the existence of complex resonance frequencies is admitted, then the existence of resonance at complex frequencies will also have to be admitted, as well as physical reality of complex frequencies as such. Then physical meaning of both complex frequencies and resonance at complex frequencies will have to be explained. And, finally, the reality of their existence will need to be proved, both theoretically and experimentally.

Existence proof of resonance at complex frequencies: Thus, it turns out that it is a matter of proof, i.e. availability or lack of it.

However, first of all, one theoretical proof of physical reality of complex frequencies has been known for a long time. It lies in the consistent and simpler interpretation of resonance at complex frequencies, compared to intricate and inconsistent interpretation of resonance at real frequencies. This circumstance fully meets the requirements outlined in the 'Occam's razor' principle.

Secondly, interpretation of resonance at complex frequencies allows explaining the inconsistencies of its interpretation at real frequencies. To this end it is possible to use Cassini ovals (Antonov 2008) and vector diagrams at complex frequencies (Antonov 2009).

In both cases it turns out that with the use of complex frequencies resonance can take place not only under the influence of continuous sinusoidal oscillations (as in the case of resonance at real frequencies), but also under the influence of exponential radio pulses (i.e. damped sinusoidal oscillations), and even exponential video pulses. The reason causing several different-value real resonance frequencies to appear is also successfully explained with the help of vector diagrams.

Thirdly, the existence of resonance at complex frequencies is proved with numerous experiments (Antonov 2008; Antonov 2009), which can be repeated by any engineer.

One of the experiments (see Fig. 1) shows that at resonance impact on an electric R,L-circuit with exponential video pulses the forced component of voltages at the resistor and the inductance turns out to have opposite signs but identical absolute value. Therefore, their sum will equal zero. This result is far from being evident, as it is well-known that in a firstorder electric circuit the phase shift between the input and output sinusoidal voltages cannot exceed $\pi / 2$. It is easy to note that this result is completely identical to resonance in the electric $\mathrm{L}, \mathrm{C}$-circuit influenced by continuous sinusoidal oscillations. As is known, forced oscillations of voltages at the inductance and the capacitor in such an L,C-circuit also turn out to be opposite in sign but equal in absolute value. Therefore, their sum will also equal zero.

And even in the electric L,C,R-circuit under resonance impact of damped sinusoidal oscillations after the transitional process is over, the sum of the forced component of voltage at the capacitor, inductance and resistor also turns out to be zero. This is an extremely interesting result, as it is impossible to get zero sum of voltages at the capacitor, inductance and resistor in such an L,C,Rcircuit impacted by continuous sinusoidal oscillations at any frequency of these oscillations. This fact alone allows making sure that true resonance takes place only at complex frequencies, whereas at real frequencies we deal only with its approximation (which can be referred to as quasi-resonance).

Yet another experiment (see Fig. 2) demonstrates that if an electric $R, L$-circuit is influenced by exponential video pulses, after the transitional process is over, the forced component of voltage in it changes its polarity to opposite with the transition of the complex frequency (i.e. in this case, the time constant) of the input action through the resonance complex frequency. And this is also 
similar to the way the forced component of voltage of an L,C-circuit impacted by continuous sinusoidal oscillations changes its polarity to opposite with the
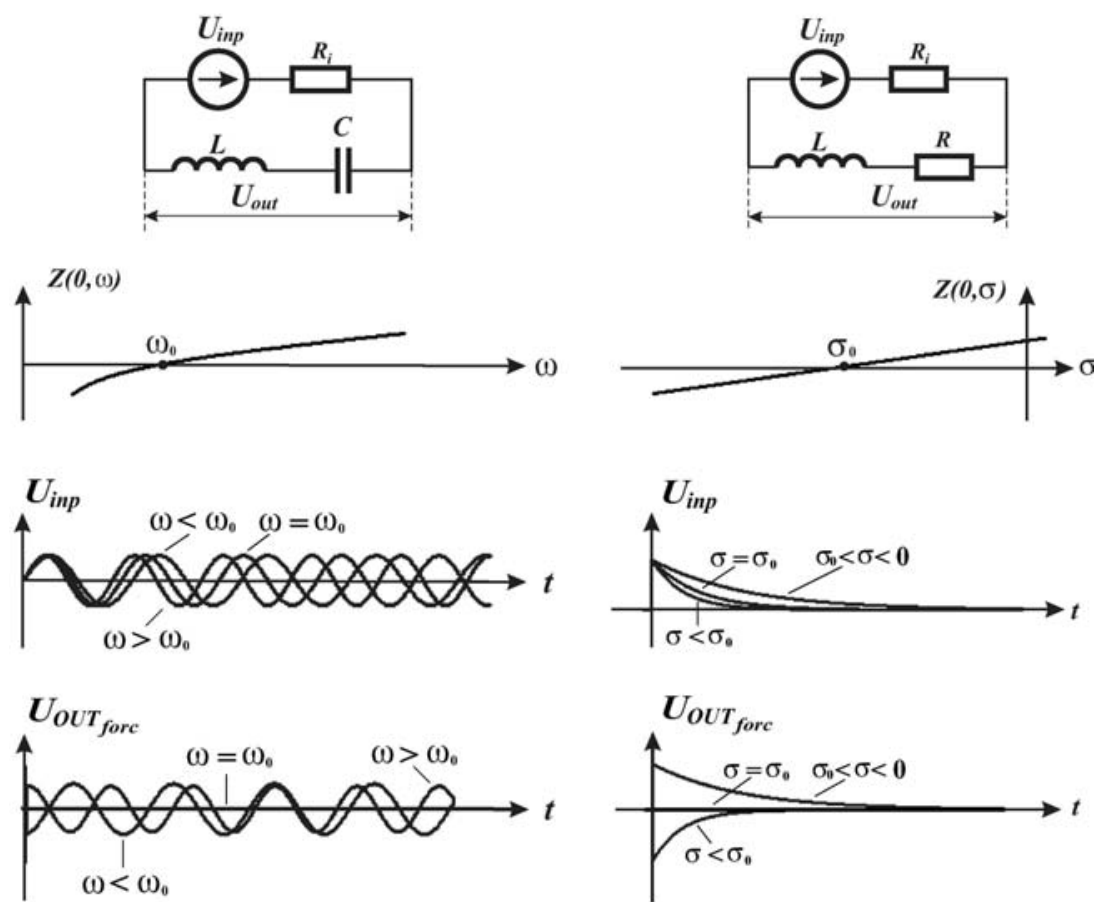

a

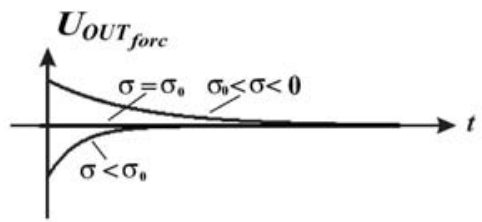

b

Fig. 1. Resonance in LC- and RL- two-pole circuits
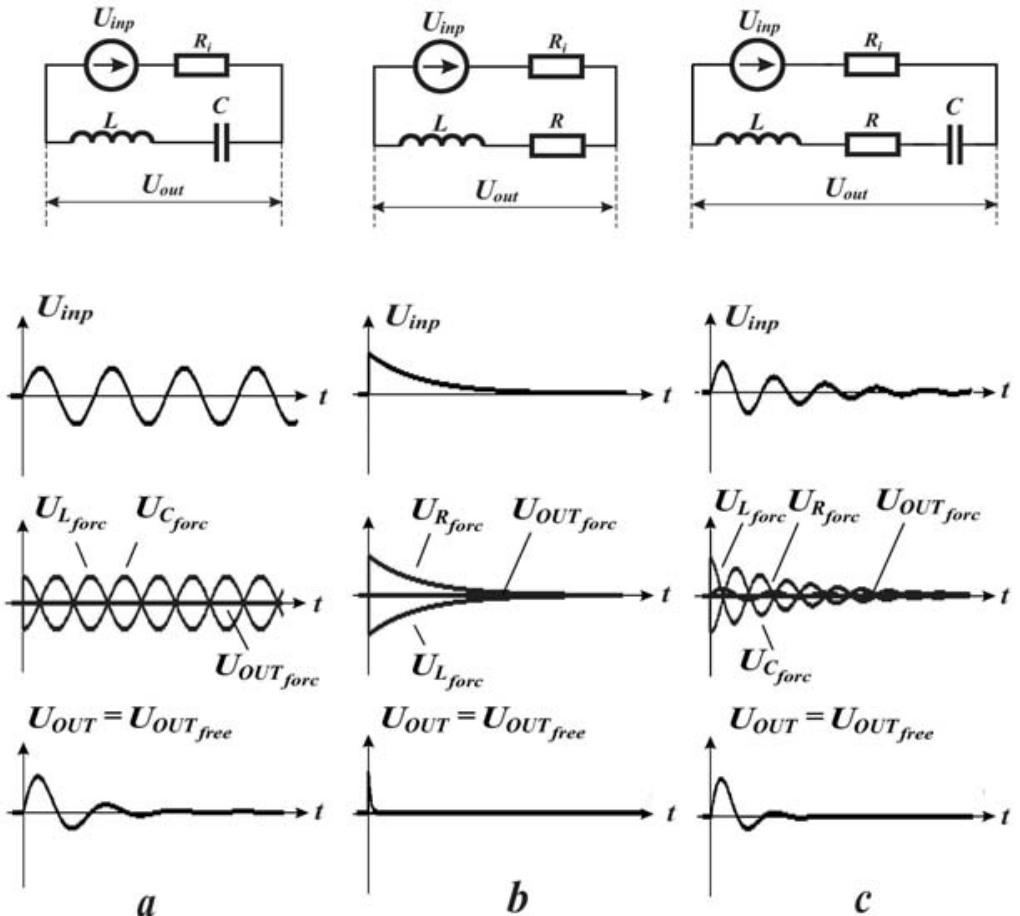

Fig. 2. Resonance in LC-, RL- and LCR-two-pole circuits 

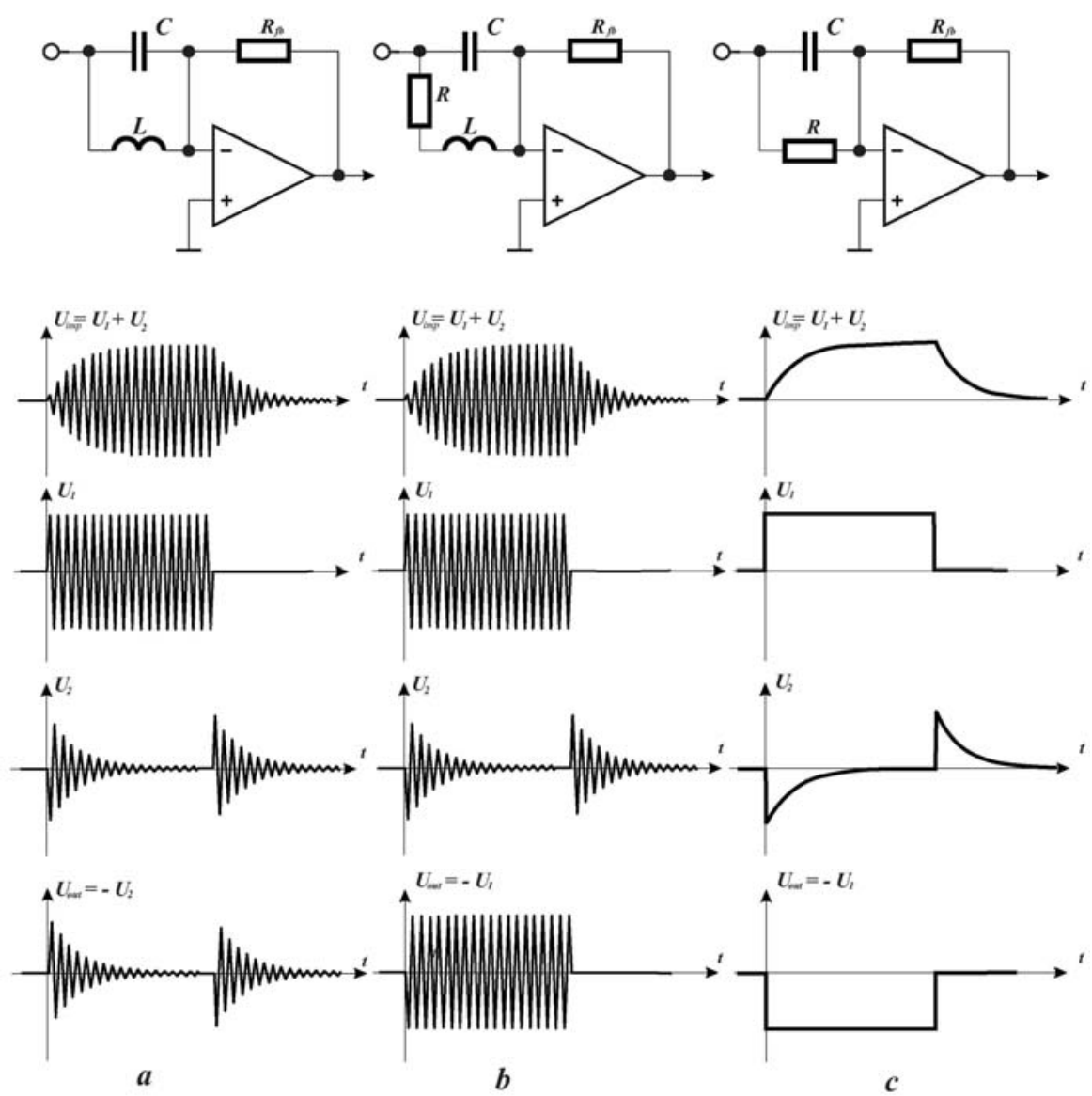

Fig. 3. Rejection filters of complex frequencies

The third experiment (see Fig. 3), which is the most convenient as special measures aimed at suppression of transitional processes need not be taken, studies electric resonance L,C-, R,C- and L,C,R-circuits. For this experiment, they are included into rejection filters. It is shown that these filters can be used to efficiently separate exponential video pulses having different values of complex frequencies (i.e. with different time constant). It is also shown that these rejection filters can be successfully used to separate exponential radio pulses (i.e. damped sinusoidal oscillations) of various complex frequencies having different real and/or imaginary component. Thus, this is a convincing proof of the fact that the notion 'complex frequency' is absolutely justified, as the selection of signals can be performed equally successfully both by real and by imaginary component of a complex frequency, as well as by both components. Let us remember here that the existing rejection filters of real frequencies make it impossible to receive such results.

We should pay attention to the fact that the latter experiment proves the possibility for developing a new class of filters - complex frequency filters, which provide for a more reliable separation of signals than the existing real frequency filters. These filters, in particular, can be used to solve the problem of electromagnetic compatibility.

Finally, Ref. (Antonov \& Bazhev 1970; Antonov 1977) give more complex patented examples of practical use of resonance at complex frequencies.

\section{Physical existence proof of concrete complex numbers}

Evidently, physical reality of complex frequencies follows from physical reality of resonance at complex frequencies. We would like to stress that, as it was mentioned above, physical reality of complex numbers can be discussed only with regard to concrete numbers. Complex frequency is in fact a concrete number.

Moreover, physical reality of complex frequency results in physical reality of complex resistances and 
conductivities, as they are a product of physically real complex frequency and some other physically real value (capacity or inductance). Further discussion would lead us to confirm physical reality of complex voltages, currents, powers and energies.

Since resonance is possible in oscillating systems having different physical nature, e.g. mechanical, complex values of the respective parameters of these oscillation processes are also physically real.

Let us note that physical reality of complex frequencies can be proven by other processes in oscillation systems, in particular, transitional processes. Here let us remember that transitional processes in oscillation systems result from changing the impact exerted on these systems, and are accounted for by the fact that parameters of forced oscillations change along with the changing impact. Thus, before and after a change of impact, energy of forced oscillations accumulated in an electric circuit is different. But energy can never change instantly, as the derivative of energy, called power, cannot be infinitely large in real physical systems. This is why transitional processes occur, which provide over the time of their duration for correlation of energy accumulated in the system before and after the impact change.

However, unlike forced oscillations which always belong to the same class of functions as the impact, transitional processes may be described by different functions. For instance, for impulse action, the transitional process may be an oscillation process. The corresponding oscillations are often referred to as impact oscillations.

In order to determine the type and parameters of transitional processes for the oscillation system under consideration by solving the differential equation, it is necessary to solve the corresponding auxiliary algebraic equation, the so called characteristic equation.

The solution of algebraic quadratic equations is taught at secondary school, and textbooks usually read that these equations have two ways of solution.

The first algorithm is as follows. If we use real numbers, then the quadratic equation $a x^{2}+b x+c=0$ depending on the correlation of values of its parameters can have either two, or one, or none solutions.

The second solution algorithm is different. According to this algorithm, the solution of the quadratic equation $a x^{2}+b x+c=0$ is found using the formula

$$
x_{1,2}=\frac{-b \pm \sqrt{b^{2}-4 a c}}{2 a}
$$

according to which, at any parameters of $a, b, c$, the equation always has two solutions. However, depending on the value of the discriminant $b^{2}-4 a c$ these solutions can be different:

- if $b^{2}-4 a c>0$ both solutions are real and different;

- if $b^{2}-4 a c=0$ both solutions are real and equal;

- if $b^{2}-4 a c<0$ both solutions are complex conjugates.

However, textbooks fail to mention that in fact one and the same quadratic equation cannot have two different mutually exclusive solutions, at least because with $b^{2}-4 a c<0$ and with the use of complex numbers the solution is considered to exist. This is why a transitional process in the corresponding electric circuit also exists and has the form of damped sinusoidal oscillations. With $b^{2}-4 a c<0$ and with the use of real numbers the solution of the characteristic equation is considered non-existent. This is why there should be no transitional process in the respective electric circuit. However, all engineers studying electric circuits know that transitional processes always exist in L,C,Rcircuits, including those in the form of damped sinusoidal oscillations. Thus, the only solution of the characteristic algebraic equation that makes sense in terms of physics is the one using complex numbers.

Thus, we once again get evidence that oscillation processes in nature are described not with real, but with complex numbers.

Then how should we interpret the solution using real numbers described above? It means that before the solution was found a condition was introduced, which did not correspond to the actual state of things in nature and stipulated that the solution should be obtained only in the form of real numbers. This is why we obtained the solution which did not correspond to the actual physical processes.

Hereinafter we shall consider the only correct solutions using complex numbers. As a human thinks with visual images, for better understanding of the 
quadratic equation's solution using complex numbers it would be useful to give some charts. However, this
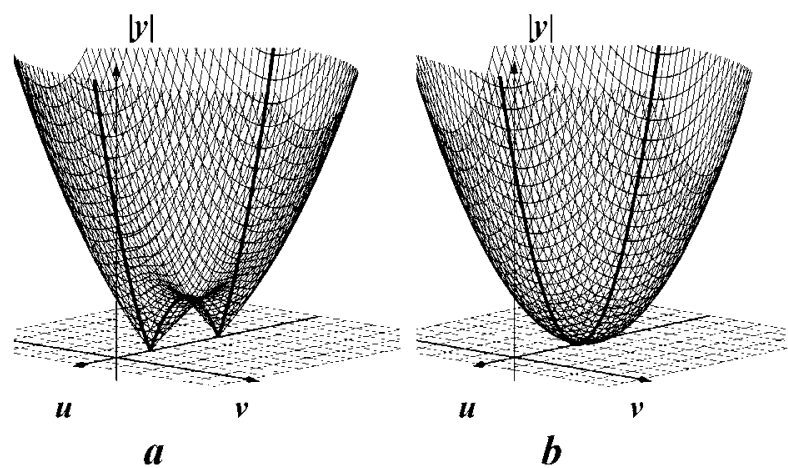

Fig. 4. Graphic solution of a quadratic equation

This obstacle can be overcome if we remember that apart from the algebraic form of representing complex figures, there are also the trigonometric and exponential forms of their representation. In accordance with them, a complex number takes zero value only at zero value of its module, irrespective of the phase value. Thus, three-dimensional space would be enough to plot the graphs, and the solution of the quadratic equation $a x^{2}+b x+c=0$ using complex numbers can be found by graphical solution of the equivalent set of equations

$$
\left\{\begin{array}{l}
|y|=\left|a x^{2}+b x+c\right|=\left|a(u+i v)^{2}+b(u+i v)+c\right| \\
|y|=0
\end{array}\right.
$$

where $|y|$ is the module of the complex variable $y$,

i.e. by finding the points of contact of the plane $|y|=0 \quad$ and the surface

$|y|=\left|a x^{2}+b x+c=a(u \pm i v)^{2}+b(u \pm i v)+c\right|$.

As is seen (Fig. 4), graphical solution in this case corresponds to the points of contact. And these two points of contact, at certain parameters of the initial quadratic equation, can be on the real axis (Fig. 4a). If the parameters $a, b, c$ of the equation change, these points can move away from each other, or they can approach each other, or even coincide (Fig. 4b). Yet at a different change of parameters $a, b, c$ of the initial algebraic equation the points of contact may move away again, but this time on the plane of complex numbers (Fig. 4c). seems to be impossible, as these charts should be four-dimensional.

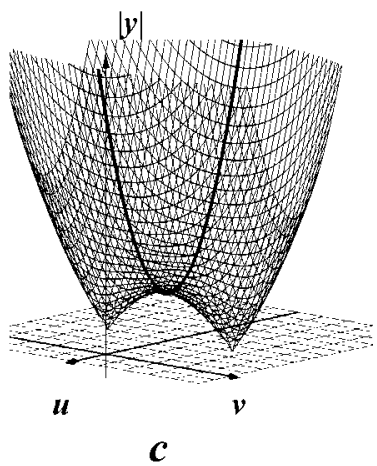

\section{CONCLUSION}

The research presented above allows formulating the new physical and mathematical principle, which suggests that concrete complex numbers the value of which depends on complex frequency are physically real. The validity of this principle has been proven theoretically and confirmed experimentally. Thus, almost 500 years of ignoring complex numbers in physics should come to an end, and search for the truth should begin.

It is easy to note, however, that many questions remained unanswered. First of all, are all other concrete complex numbers physically real, too? If they are, what processes confirm their physical reality? If not, what is their fundamental difference from physically real concrete complex numbers? Why some numbers can be complex, while others cannot? Or can all concrete numbers be complex under certain circumstances?

The new knowledge can already find practical application, for instance, in solving the problem of electromagnetic compatibility. The new knowledge can be used in developing new, better communication means.

\section{REFERENCES}

Antonov, A.A. (1987). Investigation of resonance. Preprint № 67 of the Institute of Problems of Modeling in Power Engineering of AS of the UkrSSR, Kiev.

Antonov, A.A. (2008). Physical Reality of Resonance on Complex Frequencies. European Journal of Scientific Research 21 (4), 627-641.

Antonov, A.A. (2009). Resonance on Real and Complex Frequencies. European Journal of Scientific Research 28 (2), 193-204. 
Antonov, A.A. \& Bazhev, V.M. (1970). Patent of the USSR № 433650 .

Antonov, A.A. (1977). Patent of the USSR № 879818.

Balabanian, N. (1994). Electric Circuits. NY: McGraw-Hill.

Bell, D.A. (2009). Fundamentals of Electric Circuits. Oxford, UK: Oxford University Press.

Bode, H.W. (1945). Network analysis and feedback amplifier design. Princeton, UK: Van Nostrand.

Cogdell, J.R. (1997). Foundations of Electrical Engineering. NJ: Prentice Hall.

Dolginov, A.I. (1957). Resonance in electric circuits and systems. Moscow: Gosenergoizdat.

Edminister, J. \& Nahvi, M. (1997) Schaum's Outline of Theory and Problems of Electric Circuits. NY: McGraw-Hill.

Franko, S. (1994). Electric Circuits Fundamentals. Oxford, UK: Oxford University Press.

Kantor, I.L., Solodovnikov, A.S. (1989). Hypercomplex numbers. Berlin: Springer-Verlag.

Kreyszig, E. (2006). Advanced Engineering Mathematics. NY: John Wiley \& Sons Inc.

Mandelshtam, L.I. (1955). Lectures on theory of oscillations, Vol. 4. Moscow: Publishing House of AS of the USSR.

Markushevich, A.I. (2005). Theory of Functions of a Complex Variable. American Mathematical Society.

Raymond, A.D. \& Pen-Min, L. (2001). Linear Circuit Analysis: Time Domain, Phasors, and Laplace Transforms Approaches. Oxford, UK: Oxford University Press.

Shaw, W.T. (2006). Complex Analysis with Mathematica. Cambridge University Press.

Steinmetz, C.P. (2006). Theory and Calculation of Electric Circuits. Wexford College Press.

Vinogradov, I.M., Editior-in-Chief. (1982). Mathematical Encyclopedia, Vol. 2. Moscow: Soviet Encyclopedia. 